\title{
Poly(itaconic acid) Derivatives as Thermal Stabilizers for Polystyrene and Poly(methyl methacrylate)
}

\author{
Shuzo Aoki and Atsuko FukuI \\ Department of Applied Chemistry, Faculty of Engineering, Osaka City University, \\ Sumiyoshi-ku, Osaka 558-0022, Japan
}

(Received August 20, 1997)

\begin{abstract}
The thermal degradation of a polymer blend composed of polystyrene (PSt) and poly(itaconic acid) derivatives was investigated by thermogravimetry (TG) in a nitrogen atmosphere. The temperature at the maximum rate of weight loss of the polymer bend PSt/poly(dicyclohexyl itaconate) (87/13 in weight ratio) exceeded that of each component polymer, indicating the latter polymer acted as a thermal stabilizer for PSt. Poly(itaconic anhydride) (PIAn), an intermediate product in the thermal degradation of poly(di-sec-alkyl itaconate), was prepared by the polymerization of the corresponding monomer and blended with PSt in various compositions. PIAn was found to be a highly effective stabilizer even at contents as low as $2 \%$, and it was also effective for poly(methyl methacrylate).

KEY WORDS Thermal Degradation / Poly(itaconic acid) Derivatives / Thermal Stabilizer / Polystyrene Poly(methyl methacrylate) /
\end{abstract}

The stabilization of thermoplastic resins during thermal processing is very important, and many stabilizers, consisting of low molecular weight chemicals, for preventing thermal, thermal oxidative, and photooxidative degradation of polymers are commercialized. Especially hindered phenolic antioxidants such as 2,6di-tert-butyl-4-methylphenol (BHT) are widely used to protect polymers against thermal oxidative degradation through capture of polymeric peroxy radicals (ROO-), formed in the presence of oxygen. ${ }^{1}$ However, polymeric alkyl radicals $(\mathrm{R} \cdot)$ produced in oxygen-deficient atmosphere could not be sufficiently trapped by the phenolic antioxidants such as BHT. Yachigo et al. have developed stabilizers, 2-tert-butyl-6-(3-tert-butyl-2-hydroxy-5methylbenzyl)-4-methylphenyl acrylate and 2-(1-(2-hydroxy-3,5-di-tert-pentylphenyl)ethyl)-4,6-di-tert-pentylphenyl acrylate, which are particularly effective against thermal degradation by trapping $R$ - with the olefinic double bond to give an acrylic radical followed by intramolecular hydrogen transfer from the phenolic hydroxyl group. ${ }^{2-5}$

McNeil et al. studied the degradation behavior of a $1: 1$ blend of polystyrene (PSt) and cis-1,4-polybutadiene (PBd) or polyisoprene (PIP) and found in thermogravimetry in a nitrogen atmosphere that the rate of degradation of the PSt component was markedly reduced compared with that expected in the absence of interaction between the polymers. They speculated a stabilization mechanism that the volatile decomposition products, especially 4-vinylcyclohexene from PBd and dipentene from PIP, act as inhibitors through an abstraction of allylic hydrogens with the PSt radical produced thermally. 6,7

We recently found that the blend of PSt and poly(dialkyl itaconate)s increased the degradation temperature compared with the component polymers and the copolymer composed of both monomer units in thermogravimetry in a nitrogen atmosphere. ${ }^{8}$ This suggests a means for developing a polymeric stabilizer which is expected to have improved characteristics such as no migration, non-volatility, non-toxicity, etc. The present paper deals with the details of the thermal degradation of the polymer blends of PSt and poly(dialkyl itaconate)s or their derivatives. The thermal stabilization of poly(methyl methacrylate) was also investigated in the same way.

The thermal degradation of PSt proceeds through depolymerization and other reactions. ${ }^{1,9,10}$ The reaction is initiated by random scission of the main chain giving primary and secondary radicals. The depolymerization starts and repeats from the secondary radicals by $\beta$ scission to produce the monomer molecules. The primary radicals abstract inter- and intra-molecular hydrogen atoms to stabilize themselves and leave tertiary radicals which give rise to the secondary radicals and the polymer chains with a terminal double bond by further scission of the main chain. The termination occurs monomolecularly by the evaporation of low molecular weight radicals.

The thermal degradation behavior of poly(dialkyl itaconate)s greatly differs by the kind of alkyl group. Poly(dimethyl itaconate) (PDMI) undergoes mainly depolymerization. ${ }^{11}$ Poly(di-tert-butyl itaconate) liberates the side group as isobutene and water to leave poly(itaconic anhydride) at the first stage, and then the scission of the main chain of the resulting poly(itaconic anhydride) occurs at the second stage. ${ }^{12}$ Thus primary alkyl esters depolymerize and tertiary alkyl esters decompose by liberation of the side groups. Secondary al$\mathrm{kyl}$ esters seem to be situated in a position between the primary and tertiary alkyl esters, as will be described below.

\section{EXPERIMENTAL}

PSt and poly(methyl methacrylate) (PMMA) were obtained by thermal polymerization of the purified monomer without added radical initiators. Poly(dialkyl itaconate)s were prepared by polymerization initiated by azobisisobutyronitrile from the monomers obtained by the reaction of itaconic anhydride with the corresponding alcohols, ${ }^{13}$ except PDMI. PDMI and poly(itaconic 
Table I. Molecular weight and polydispersity of polymer samples

\begin{tabular}{lcc}
\hline Polymer & $\bar{M}_{n} \times 10^{-4}$ & $\bar{M}_{w} / \bar{M}_{n}$ \\
\hline PSt & 36.2 & 1.5 \\
PDMI & 0.36 & 1.8 \\
PDCHI & 8.4 & 2.3 \\
PDiPI & 6.4 & 1.8 \\
PIAn $^{\mathrm{a}}$ & 2.0 & 1.4 \\
PMMA & 185 & 1.3 \\
\hline
\end{tabular}

${ }^{a}$ Determined for dimethyl ester.

anhydride) were prepared from the monomers obtained commercially, similarly to the other polymers. ${ }^{14}$ These polymers were purified by reprecipitation and dried thoroughly in vacuo. The number average molecular weight $\left(\bar{M}_{n}\right)$ and polydispersity $\left(\bar{M}_{w} / \bar{M}_{n}\right)$ of the polymer samples were determined by gel-permeation chromatography using polystyrene standard and are tabulated in Table I. Other polymer samples used were styrene/maleic anhydride alternating copolymer prepared by radical copolymerization of equimolar amounts of styrene and maleic anhydride in toluene, isobutene/maleic anhydride alternating copolymer which was a commercial product provided by Kuraray Co., and poly(dicyclohexyl fumarate) which was offered by NOF Corp. Naphthalic anhydride (Tokyo Kasei) was recrystallized from ethanol.

The preparation of the polymer blend was carried out by dissolving two polymer samples in a common good solvent and pouring the solution into a non-solvent to precipitate the polymer mixture, followed by filtration and drying in vacuo. As a reference composition of the polymer blend, $87 \%$ PSt was taken unless otherwise noted.

Thermogravimetry (TG) was performed using a Rigaku Thermoflex TG 8110 under a nitrogen atmosphere at a heating rate of $10^{\circ} \mathrm{Cmin}^{-1}$. The simulated TG curve of the polymer blend was obtained from the initial polymer blend ratio and the results of TG experiments of the component polymers performed separately. Glass transition temperature $\left(T_{\mathrm{g}}\right)$ was determined by differential scanning calorimetry (DSC) using a Seiko SSC-5000. The pyrolysis-gas chromatography/ mass spectrometry (Py-GC/MS) experiments were performed by using a Hewlett Packard 5890 II Gas chromatograph equipped with a pyrolyzer (Nippon Bunseki Kogyo JHP-3) and a Nippon Denshi JMS-BU20 mass spectrometer. The pyrolysis of the polymer was carried out at the set temperature of $590^{\circ} \mathrm{C}$ under a helium atmosphere.

\section{RESULTS AND DISCUSSION}

\section{PSt/Poly(dialkyl itaconate)s}

TG measurements of PSt and PDMI showed that PSt is significantly more stable than PDMI, as shown in Figure 1. The stability of the blend of both polymers was slightly lower than that simulated from the results of both components, indicating the absence of a positive synergistic effect. The TG curve of poly(dicyclohexyl itaconate) (PDCHI) showed that the temperature of the maximum rate of weight loss $\left(T_{\max }\right)$ was lower than that of PDMI (Figure 2). The TG curve of the polymer blend,

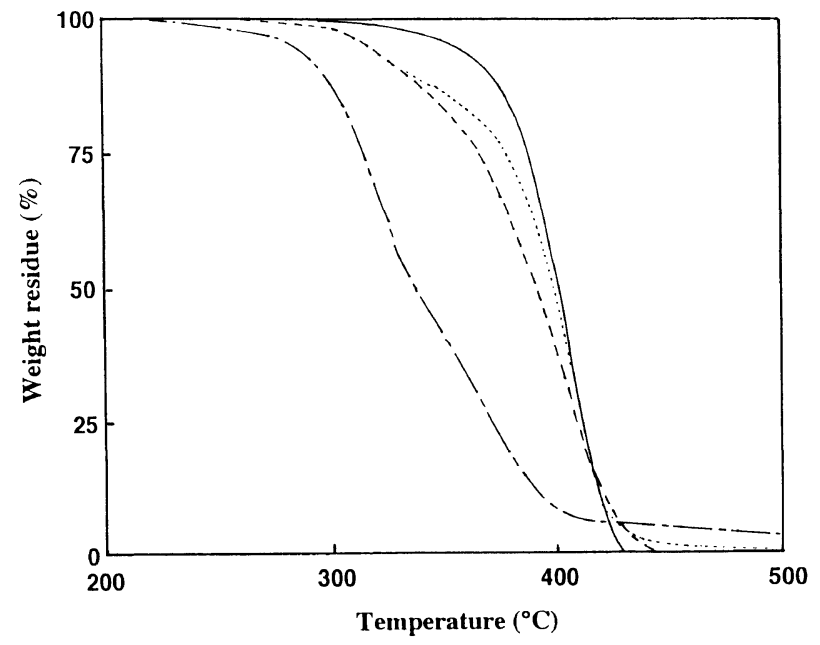

Figure 1. TG curves for PSt (-), PDMI (-- - ), blend of PSt/PDMI in $87 / 13$ wt. ratio $(---)$, and the simulated curve for the polymer blend (-----).

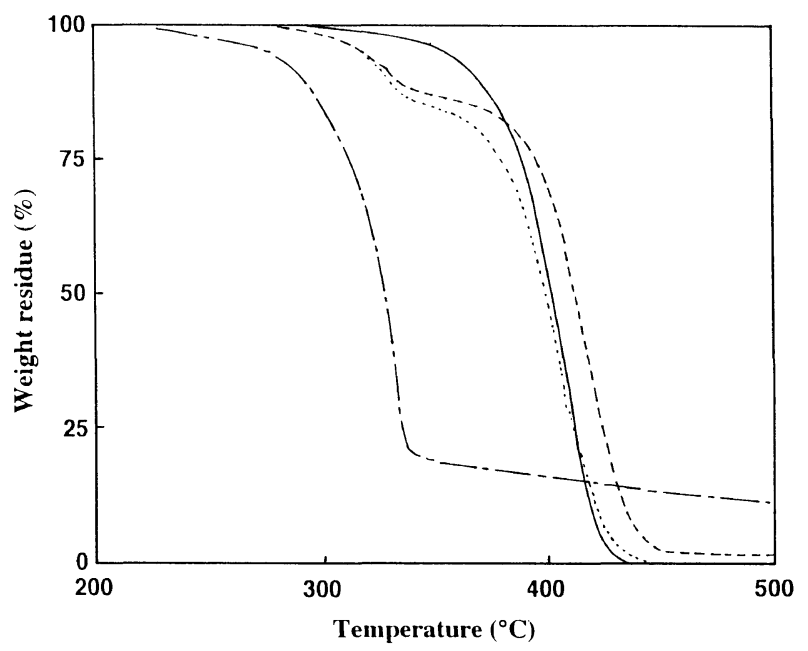

Figure 2. TG curves for PSt $(-)$, PDCHI $(---)$, blend of PSt/PDCHI in $87 / 13 \mathrm{wt}$. ratio (---), and the simulated curve for the polymer blend (-----).

Table II. TG data of PSt, PDMI, PDCHI, PIAn, PMMA, and the polymer blends

\begin{tabular}{|c|c|c|c|c|}
\hline \multirow{2}{*}{ Polymer } & \multirow{2}{*}{$\begin{array}{c}\text { Weight } \\
\text { ratio }\end{array}$} & \multirow{2}{*}{$\frac{T_{\text {init }}}{{ }^{\circ} \mathrm{C}}$} & \multirow{2}{*}{$\frac{T_{\max }}{{ }^{\circ} \mathrm{C}}$} & \multirow{2}{*}{$\frac{\text { Residue at } 500^{\circ} \mathrm{C}}{\%}$} \\
\hline & & & & \\
\hline PSt & 100 & 298 & 413 & 0 \\
\hline PDMI & 100 & 205 & 371 & 3.9 \\
\hline PSt/PDMI & $87 / 13$ & 263 & 407 & 0 \\
\hline PDCHI & 100 & 233 & 334 & 11.7 \\
\hline PSt/PDCHI & $87 / 13$ & 273 & 419 & 1.9 \\
\hline PIAn & 100 & 64 & - & 39.1 \\
\hline PSt/PIAn & $87 / 13$ & 158 & 447 & 7.1 \\
\hline PMMA & 100 & 219 & 362 & 0 \\
\hline PMMA/PIAn & $95 / 5$ & 184 & 381 & 4.7 \\
\hline
\end{tabular}

PSt/PDCHI, showed two-stage degradation behavior, and the $T_{\max }$ in the second stage exceeded that of PSt, in spite of the fact that the simulated TG curve of the polymer blend is located between those of PSt and PDCHI. These results are summarized in Table II. The thermal degradation behavior of the polymer blend, PSt/PDCHI, especially in the second stage, was partic- 


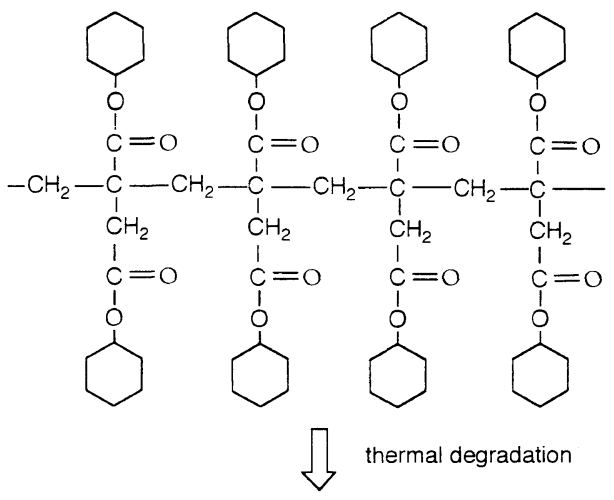

depolymerization

side-group liberation and anhydride ring formation
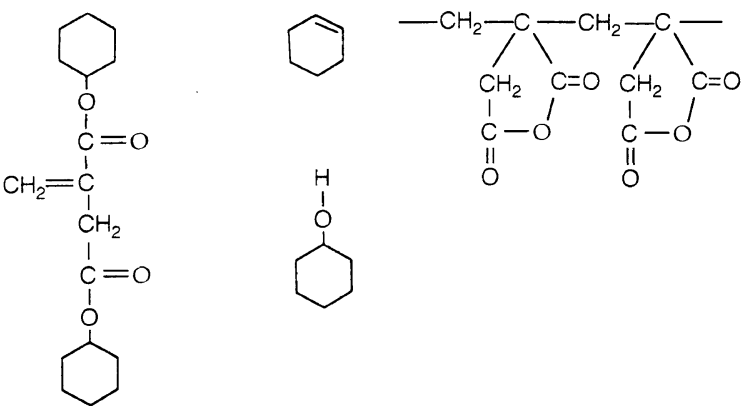

Scheme 1. Thermal degradation of poly(dicyclohexyl itaconate)

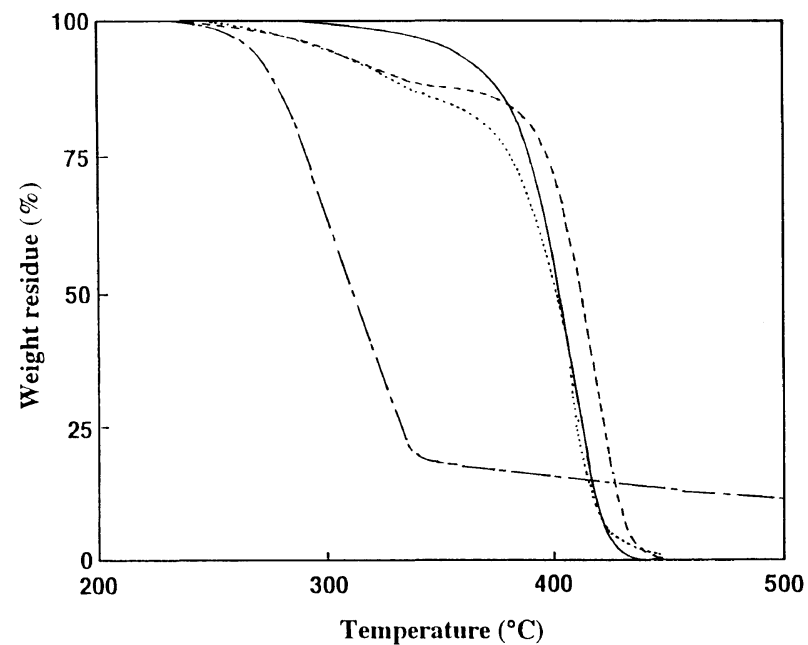

Figure 3. TG curves for PSt (-), PDiPI (-- - ), blend of PSt/PDiPI in $87 / 13 \mathrm{wt}$. ratio (--), and the simulated curve for the polymer blend (------).

ularly interesting in studying the stabilization.

The thermal degradation of PDCHI, a secondary alkyl ester, proceeds simultaneously through the depolymerization process giving dicyclohexyl itaconate monomer and the side-group liberation process producing volatile compounds, such as cyclohexene and cyclohexanol, and forming the anhydride ring structure (Scheme 1). ${ }^{14}$ The results of the TG of PSt/PDCHI blend indicated that PDCHI acts as a thermal stabilizer for PSt. The blend of PSt and poly(diisopropyl itaconate) (PDiPI) gave similar results to those of PSt/PDCHI, as shown in Figure 3. The thermal degradation process of PDiPI seems analogous to PDCHI, because it is also a secondary alkyl ester. Contribution of the depolymerization process for the PSt stabilization is ruled out from the results of PDMI

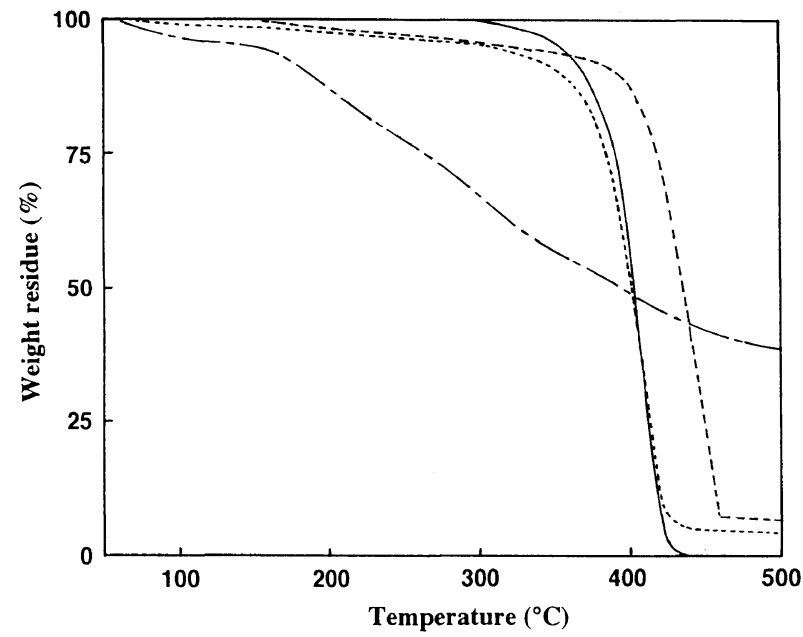

Figure 4. TG curves for PSt (- - , PIAn (-- - ), blend of PSt/PIAn in $87 / 13 \mathrm{wt}$. ratio (-- ), and the simulated curve for the polymer blend (-----).

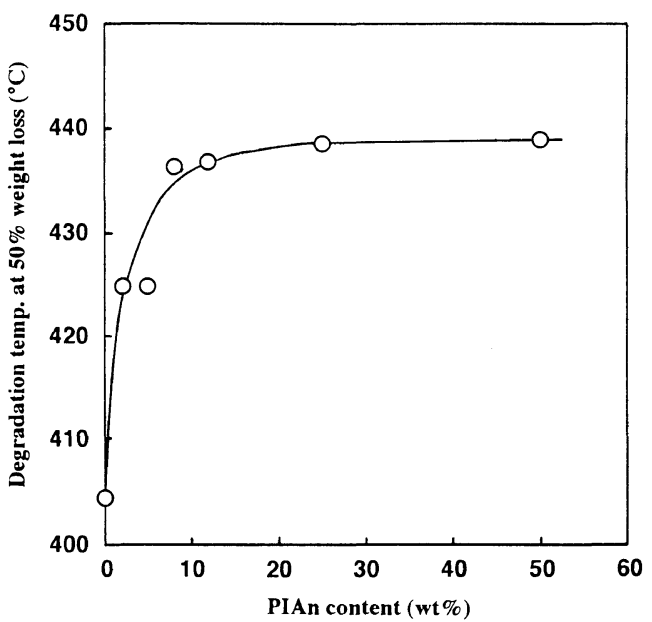

Figure 5. Effects of PSt/PIAn composition on thermal stability of the polymer blend.

which degrades mainly through the depolymerization. ${ }^{11}$ This was also confirmed in the TG experiments of the blend of PSt and poly(methyl methacrylate) which is an exclusively depolymerizable polymer. Thus, the sidegroup liberation process, especially the formation of poly(itaconic anhydride) (PIAn), appears responsible for the stabilization effect.

\section{PSt/Poly(itaconic anhydride)}

To clarify the participation of PIAn in the stabilization of PSt, the TG experiments of the polymer blend, PSt/PIAn, were carried out. The results are shown in Figure 4. The temperature of initiation of the weight loss $\left(T_{\text {init }}\right)$ of PIAn was below $100^{\circ} \mathrm{C}$. This is significantly lower than those for PDMI and PDCHI $\left(>200^{\circ} \mathrm{C}\right)$. Above the $T_{\text {init }}$, the degradation proceeded gradually and left a large quantity of residue even at $500^{\circ} \mathrm{C}$. The TG experiments of the polymer blend, PSt/PIAn, gave lower $T_{\text {init }}$ than PSt due to the low $T_{\text {init }}$ of PIAn, while the stability, especially above $400^{\circ} \mathrm{C}$, increased to significantly exceed that of PSt . When the degradation temperature at $50 \%$ weight loss was taken as a measure of relative stability, the effect of the content of PIAn in the polymer blend given in Figure 5 showed that PIAn 


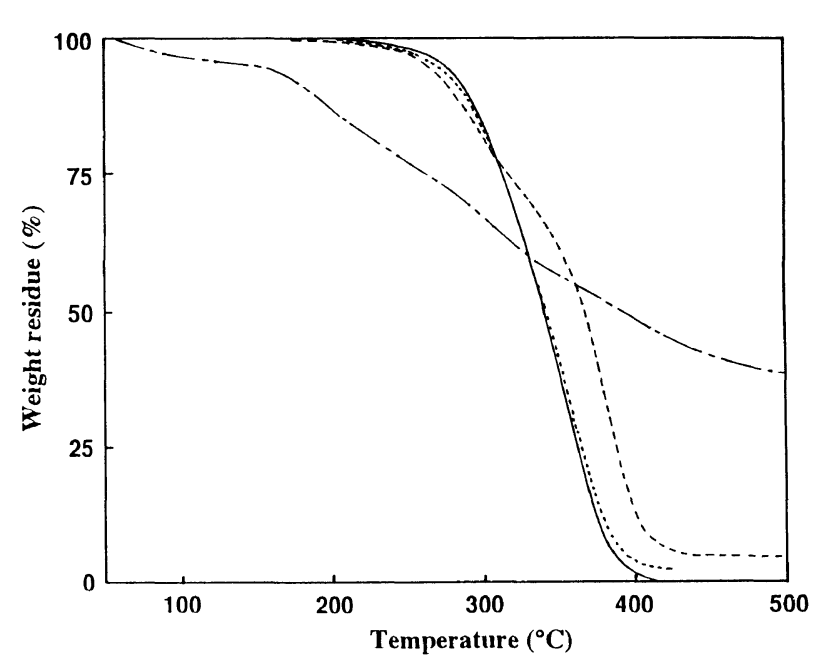

Figure 6. TG curves for PMMA (-), PIAn (-- - ), blend of PMMA/PIAn in $95 / 5$ wt. ratio (--), and the simulated curve for the polymer blend (------).

was an effective thermal stabilizer for PSt even at contents as low as $2 \%$. Thus, the active species providing the stabilization effect in the TG experiments of the polymer blend, PSt/PDCHI, was confirmed to be PIAn produced as an intermediate product of degradation.

\section{PMMA/Poly(itaconic anhydride)}

TG measurements of PMMA and the polymer blend, PMMA/PIAn (95/5 in weight ratio), were performed. The results are shown in Figure 6. PMMA degraded at lower temperature than PSt (Table II). The TG curve of the polymer blend showed two-step degradation having an inflection point at $c a .310^{\circ} \mathrm{C}$. The $T_{\text {init }}$ and the stability below the inflection point decreased due to the contribution of the very low $T_{\text {init }}$ of PIAn, but stability above the inflection point greatly exceeded that of PMMA, indicating PIAn acts as a stabilizer similar to the case of PSt/PIAn. The presence of the inflection point suggests that PIAn is effective as the stabilizer above this temperature. In the case of PSt/PIAn, the inflection point was not observed probably due to higher $T_{\text {init }}$ of PST.

\section{Effects of Other Anhydrides}

If the stabilization effect of PIAn is due to the presence of an anhydride ring, other polymeric and monomeric anhydrides might also be effective as PSt stabilizers. To prove this, attempts were carried out using styrene/ maleic anhydride alternating copolymer, isobutene/ maleic anhydride alternating copolymer, poly(dicyclohexyl fumarate) which leaves an anhydride residue during thermal degradation just as PDCHI, ${ }^{15}$ and naphthalic anhydride. None of these anhydrides showed any stabilizing activity for PSt degradation. Consequently, the stabilizing activity is concluded not to be only due to the anhydride ring structure. PIAn seems to be rather specific in its activity. The copolymer of St and DCHI as a precursor of itaconic anhydride (IAn) unit did not have thermal stability exceeding that of PSt. ${ }^{8}$ This indicates the requirement for a certain length of the IAn unit sequence.

\section{Miscibility of Polymer Blend}

PSt and PMMA effectively stabilized by PIAn degrade thermally through the depolymerization process by radical mechanism. Therefore, PIAn seems to break the radical chain. There are two possibilities for the stabilization function of PIAn; PIAn itself and its degradation fragments. If PIAn itself reacts with a polymer radical thermally produced to deactivate it, it would be affected by miscibility of PIAn and PSt or PMMA. $T_{\mathrm{g}}$ measurement by DSC and the opacity of the melt pressed sheet showed that PSt is not miscible with PDMI, PDCHI, and PIAn, and also PMMA with PIAn. Therefore, the mechanism that PIAn directly acts as the stabilizer is rather questionable.

\section{Degradation Fragments in Thermal Decomposition of PIAn}

The radical species as intermediate products in the thermal degradation of PIAn are presumed to react with the polymer radical of PSt and PMMA produced also at the same time in the thermal degradation to terminate the chain reaction. An analysis of the degradation fragments of PIAn by means of Py-GC/MS was attempted. The presence of monomeric IAn and carbon dioxide in the fragments was confirmed by MS, indicating that both depolymerization and side-group liberation occurred. However, further information could not be obtained. Thus, the mechanism and the structure of the active species for polymer stabilization are still not clear.

\section{CONCLUSIONS}

The following results were obtained in the present work.

(1) The thermal stability of PSt was improved in the presence of poly(di-sec-alkyl itaconate) in a nitrogen atmosphere.

(2) Poly(itaconic anhydride) was found to be highly effective for the PSt stabilization even at contents as low as $2 \%$ and was concluded to be the active species providing the stabilization effect in the polymer blend, PSt/poly(di-sec-alkyl itaconate), because it was an intermediate product in the thermal degradation of poly(di-sec-alkyl itaconate).

(3) Poly(itaconic anhydride) also showed stabilizing activity for PMMA.

Acknowledgment. The authors thank Wakayama Industrial Technology Center for permission to use TG and Py-GC/MS instruments. We also thank Messrs. Ch. Nakaoka, M. Takagaki, and A. Matsumoto for valuable comments.

\section{REFERENCES}

1. N. L. Maecker and D. N. Armentrout, "Encyclopedia of Polymer Science and Engineering," Vol. 16, John Wiley \& Sons, New York, N.Y., 1989, p 180.

2. S. Yachigo, M. Sasaki, Y. Takahashi, F. Kojima, T. Tanaka, and T. Okita, Polym. Degrad. Stab., 22, 63 (1988).

3. S. Yachigo, F. Kojima, M. Sasaki, K. Ida, S. Tanaka, and K. Inoue, Polym. Degrad. Stab., 37, 107 (1992).

4. S. Yachigo, K. Ida, M. Sasaki, K. Inoue, and S. Tanaka, Polym. Degrad. Stab., 39, 317 (1993). 
5. S. Yachigo, M. Sasaki, K. Ida, K. Inoue, S. Tanaka, Y. Honda, E. Fukuyo, and K. Yanagi, Polym. Degrad. Stab., 39, 329 (1993).

6. I. C. McNeil, L. Ackerman, and S. N. Gupta, J. Polym. Sci., Polym. Chem. Ed., 16, 2169 (1978).

7. I. C. McNeil and S. N. Gupta, Polym. Degrad. Stab., 2, 95 (1980).

8. S. Mori, A. Fukui, S. Aoki, and T. Otsu, Polym. Prepr., Jpn., 44, 270, 1368 (1995).

9. W. Schnabel, "Polymer Degradation-Principles and Practical Applications," Hanser International, München, 1981, p 25.
10. A. Guyot, Polym. Degrad. Stab., 15, 219 (1986).

11. J. Veličković and I. Popović, Croatia Chem. Acta, 60, 173 (1987).

12. T. Otsu and H. Watanabe, Eur. Polym. J., 29, 167 (1993).

13. J. Z. Yang, O. Nakatsuka, and T. Otsu, Chem. Express, 5, 805 (1990).

14. M. Fujishima, MS thesis, Osaka City University, Osaka, Japan, 1994.

15. S. Aoki and A. Fukui, unpublished results. 